Available online on 15.12.2020 at http://jddtonline.info
OC 2011-20, publisher and licensee JDDT, This is an Open Access article which permits
unrestricted non-commercial use (CC By-NC), provided the original work is properly cited

Open 1 Access

\title{
Coronavirus: A Disease of the Haemoglobin? Suggested Therapeutic Approaches
}

\author{
Diedhiou Moustapha $1^{*}$, Dieye A. ${ }^{2}$, Makalou D. ${ }^{3}$, Ba E.B. ${ }^{4}$, Fall M.L. ${ }^{1}$, Dia N.M. ${ }^{2}$ \\ 1: Anesthesia-Resuscitation Department - Saint Louis Regional Hospital / Faculty of medicine/ Gaston Berger University / Senegal \\ 2: Infectious Diseases Department - Saint Louis Regional Hospital / Faculty of medicine/ Gaston Berger University / Senegal \\ 3: Biology laboratory - Saint Louis Regional Hospital / Faculty of medicine/ Gaston Berger University / Senegal \\ 4: Anesthesia-Resuscitation Department - Fann Hospital / Faculty of medicine/ Cheikh Anta Diop University / Senegal
}

\begin{abstract}
The pathophysiology of the coronavirus infection is not yet fully understood. Several theories have been developed from the acute respiratory distress syndrome to antiphospholipid antibody syndrome to vascular thrombosis. It is in this context that we propose through brainstorming some proposals for the therapeutic management based on secondary haemoglobin damage by COVID-19.
\end{abstract}

Keywords: coronavirus - haemoglobin - chloroquine - pathophysiology

Article Info: Received 11 Oct 2020; $\quad$ Review Completed 17 Nov 2020; $\quad$ Accepted 22 Nov 2020; Available online 15 Dec 2020

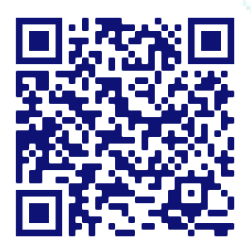

Cite this article as:

Diedhiou M, Dieye A, Makalou D, Ba EB, Fall ML, Dia NM, Coronavirus: A Disease of the Haemoglobin? Suggested

Therapeutic Approaches, Journal of Drug Delivery and Therapeutics. 2020; 10(6-s):1-2

http://dx.doi.org/10.22270/jddt.v10i6-s.4405

*Address for Correspondence:

Diedhiou Moustapha, Anesthesia-Resuscitation Department - Saint Louis Regional Hospital / Faculty of medicine/ Gaston Berger University / Senegal

\section{Dear Editor:}

The therapeutic management of coronavirus infection is based on symptomatic treatment ${ }^{1}$. Treatment proposals are made with more or less satisfactory results. The proposed therapies are diverse and varied and include: corticosteroid therapy 2 , antivirals or antiretrovirals or 3 , chloroquine or hydroxychloroquine ${ }^{4}$.... Indeed, only the latest (chloroquine) seems to give good results in terms of improving the cure rate and reducing the length of hospitalization ${ }^{4}$. It is in this global context that we have carried out this work, the aim of which is to offer readers the new therapeutic and management prospects for COVID 19 that are being considered in the light of the new pathophysiological data on the coronavirus.

The coronavirus infection has not finished revealing all its facets. Initially (December 2019) all the theories have evoked an alveolar-capillary damage by the virus leading to an acute respiratory distress syndrome (ARDS) and cellular hypoxia or anoxia which if not treated evolves towards Multivisceral Failure (MVF) and death 5. Currently (April 2020), new pathophysiological theories are emerging and are based on feedback from deceased and cured cases. Three situations are then observed through the scientific literature: death of patients previously under invasive ventilation due to ARDS and/or multivisceral failure; total recovery (or at least a drop in viral load to be exact); readmission of patients for tiredness and/or post hypoxic or anoxic encephalopathy. Indeed, these situations have made it possible to understand that the respiratory distress of COVID-19 patients is not similar to that observed during ARDS (Acute Respiratory Distress Syndrome) and/or pneumonia, where the primary cause is the destruction of the alveolar-capillary membrane (ARDS) or the immune reaction to a pathogenic germ (pneumonia). Patients who still show signs of tiredness and post-hypoxic leukoencephalopathy after being declared cured justify that the main problem is not pulmonary and/or respiratory. Moreover, hemolysis had already reported in patients with SARS (coronavirus from $2003-2004)^{6}$.

According to new pathophysiological data, the COVID-19 virus, after crossing the alveolar-capillary barrier, attacks red blood cells and their haemoglobin and releases the iron molecule they contain 7 . The coronavirus denatures and destroys porphyrin, which is a molecule within the haem and which encompasses the iron atom 7 .

The main role of the iron atom is to bind to oxygen and thus allow its transport. The end result of porphyrin destruction is an increase in circulating iron, oxidative stress and iron overload when immune defence mechanisms are overwhelmed. The haemoglobin molecules therefore 
become physiologically inactive and no longer play their role as oxygen carriers 7 . The following situations then occur: excess iron in the lungs (in the alveolar-capillary membrane) and in the body; lactic acidosis secondary to hypoxia or anoxia, multivisceral failure due to hypoxia or cellular anoxia. It is this massive release of iron that explains the spectacular ground- glass images observed on thoracic CT 7 . These images are in fact a reflection of the atomic metal of the iron exposed as a result of the aggression of the red blood cell by the coronavirus. The generalized oxygen debt in all organs explains the diversity of clinical situations encountered (muscle and joint pain, dyspnea, anosmia, digestive signs ...) 8 .

Moreover, if chloroquine treats malaria and seems to have an effect on the evolution of the coronavirus, it is because the two infections probably have a pathophysiological point in common. This common factor is linked to the fact that the germs, the plasmodium on the one hand and the coronavirus on the other hand, penetrate the red blood cells. Indeed, one of the actions of chloroquine on Plasmodium falciparum is that it "crosses the membrane of the red blood cell and then that of Plasmodium and penetrates into the vacuole where they accumulate" 9 . It then promotes the destruction of the plasmodium. By the same mechanism in patients with coronavirus, chloroquine then crosses the membrane of the red blood cells of these patients. The exact mechanisms of action of chloroquine within hemoglobin are not well known at this time, but we can put evoke the hypothesis that chloroquine participates in a modification of the physicochemical environment within red blood cells, thus preventing or reducing the action of the coronavirus towards the haem. It is not fully effective against coronavirus in in vitro tests 10 ; however, by modifying the physicochemical environment of the red blood cells in which the coronavirus is found, it reduces or prevents its action. Chloroquine then promotes the maintenance of arterial oxygen transport by haemoglobin and thus prevents multivisceral failure. These effects of chloroquine are then more than beneficial for the body's defence systems, which can then be deployed to eradicate the virus.

Finally, these new pathophysiological data make it possible to redefine new therapeutic perspectives or objectives. In the absence of an effective vaccine or virucidal treatment, the therapeutic objectives will be the improvement of arterial oxygen transport (by oxygen therapy, transfusion and/or optimization of cardiac output), the improvement of the immune system (rehydration, diet, vitamin $\mathrm{C}$, etc.), the fight against iron-related oxidative stress (iron chelator, etc.) and symptomatic treatment.

The principle of mechanical ventilation applied in coronavirus patients is that of ventilation for ARDS 11. This means applying positive expiratory pressure, small volume, high frequency... This principle is completely at odds with the above-described pathophysiology because here the disease is not pulmonary but rather pathology of the erythrocyte, of the haemoglobin. Ventilation could moreover worsen the lungs of these patients because of the positive pressure. So as an alternative therapy against coronavirus, the same therapeutic principles should be adopted as for patients who are hypoxic at altitude and/or carbon monoxide poisoning or haemoglobinopathies. The oxygen debt observed during coronavirus is secondary to a decrease in arterial oxygen transport $(\mathrm{TaO} 2=\mathrm{CaO} 2 \times \mathrm{Q}=\mathrm{Hb} \times \mathrm{SaO} 2 \times$ $\mathrm{Q} \times$ math constant). To improve this transport, in this context of no decrease in cardiac output, it is sufficient to increase oxygen saturation and to transfuse the patients in order to improve the physiologically active circulating hemoglobin. Indeed, it is necessary to:

-Maintain non-invasive oxygen therapy for as long as possible; give preference to cautious NIV (non-invasive ventilation) using filters that prevent aerosolization of the virus. Hyperbaric oxygen therapy proposed by some authors is an alternative that is difficult to achieve in this context of massive influx of patients in hospitals.

-Same blood group and rhesus group blood transfusion even if the count does not show anaemia when the oxygen saturation is very low with signs of poor tolerance.

-Think about administering iron chelators to reduce oxidative stress and side effects related to iron accumulation in the body.

Serum Iron test could with this new data be useful in the diagnosis and monitoring of therapeutic efficacy. Indeed, with a double safety effect, the correlation between serum iron level and viral load could allow the detection of false positives or false negatives. Monitoring of the serum iron curve could be useful in monitoring therapeutic efficacy. In sub-Saharan Africa, where there are not many laboratories capable of screening for coronavirus, serum iron testing for disease progression monitoring is a relevant alternative.

Finally, future studies on covid-19 and haemoglobinopathies will undoubtedly explain the still low prevalence of coronavirus and severe forms in sub-Saharan Africa where there is a high incidence of haemoglobin-related pathologies.

\section{Conflict of interest statement: none}

\section{References:}

(1) PREUX, Pierre-Marie. Coronavirus: "We speak rather of a moderate virus". 2020

(2) Pariente, R. So-called atypical coronavirus pneumopathies. CMEPneumology, 2004; 1(1):3-6

(3) Beck, B. R., Shin, B., Choi, Y., Park, S., \& Kang, K. Predicting commercially available antiviral drugs that may act on the novel coronavirus (2019-nCoV), Wuhan, China through a drug-target interaction deep learning model. bioRxiv. 2020.

(4) Colson, P., Rolain, J. M., \& Raoult, D. Chloroquine for the 2019 novel coronavirus. Int J Antimicrob Agents. 2020.

(5) World Health Organization, O. Clinical management of severe acute respiratory infection when infection with a new coronavirus (nCoV) is suspected: interim guidelines, 25 January 2020 (No. WHO/nCoV/Clinical/2020.2). World Health Organization. 2020.

(6) Pariente, R. So-called atypical coronavirus pneumopathies. CMEPneumology, 2004; 1(1):3-6.

(7) Liu, W., \& Li, H. COVID-19: Attacks the 1-Beta Chain of Hemoglobin and Captures the Porphyrin to Inhibit Human Heme Metabolism.

(8) Storz, J., Stine, L., Liem, A., \& Anderson, G. A. Coronavirus isolation from nasal swab samples in cattle with signs of respiratory tract disease after shipping. Journal of the American Veterinary Medical Association, 1996; 208(9):1452-1455.

(9) Mungthin, M., Bray, P. G., Ridley, R. G., \& Ward, S. A. Central role of hemoglobin degradation in mechanisms of action of 4aminoquinolines, quinoline methanols, and phenanthrene methanols. Antimicrobial agents and chemotherapy, 1998; 42(11):2973-2977.

(10) Cortegiani, A., Ingoglia, G., Ippolito, M., Giarratano, A., \& Einav, $\mathrm{S}$. A systematic review on the efficacy and safety of chloroquine for the treatment of COVID-19. Journal of critical care. 2020.

(11) Wu, C., Chen, X., Cai, Y., Zhou, X., Xu, S., Huang, H., ... \& Song, J. Risk factors associated with acute respiratory distress syndrome and death in patients with coronavirus disease 2019 pneumonia in Wuhan, China. JAMA internal medicine. 2020. 